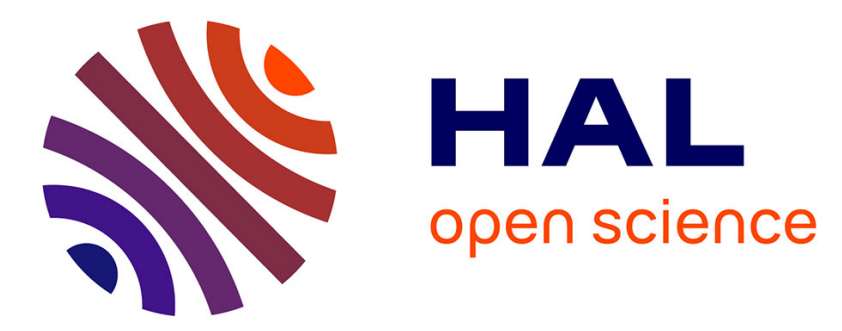

\title{
Rheology of granular materials composed of nonconvex particles
}

\author{
Baptiste Saint-Cyr, Jean-Yves Delenne, Charles Voivret, Farhang Radjai, \\ Philippe Sornay
}

\section{- To cite this version:}

Baptiste Saint-Cyr, Jean-Yves Delenne, Charles Voivret, Farhang Radjai, Philippe Sornay. Rheology of granular materials composed of nonconvex particles. Physical Review E: Statistical, Nonlinear, and Soft Matter Physics, 2011, 84, pp.041302. 10.1103/PhysRevE.84.041302 . hal-00759629

\section{HAL Id: hal-00759629 \\ https://hal.science/hal-00759629}

Submitted on 27 Apr 2021

HAL is a multi-disciplinary open access archive for the deposit and dissemination of scientific research documents, whether they are published or not. The documents may come from teaching and research institutions in France or abroad, or from public or private research centers.
L'archive ouverte pluridisciplinaire HAL, est destinée au dépôt et à la diffusion de documents scientifiques de niveau recherche, publiés ou non, émanant des établissements d'enseignement et de recherche français ou étrangers, des laboratoires publics ou privés. 


\title{
Rheology of granular materials composed of nonconvex particles
}

\author{
B. Saint-Cyr, ${ }^{1,2}$ J.-Y. Delenne, ${ }^{1}$ C. Voivret, ${ }^{1}$ F. Radjai, ${ }^{1}$ and P. Sornay ${ }^{2}$ \\ ${ }^{1}$ LMGC, CNRS-Université Montpellier 2, Place Eugène Bataillon, F-34095 Montpellier cedex 05, France \\ ${ }^{2}$ CEA, DEN, SPUA, LCU, F-13108 Saint Paul lez Durance, France
}

(Received 19 April 2011; published 10 October 2011)

\begin{abstract}
By means of contact dynamics simulations, we investigate the shear strength and internal structure of granular materials composed of two-dimensional nonconvex aggregates. We find that the packing fraction first grows as the nonconvexity is increased but declines at higher nonconvexity. This unmonotonic dependence reflects the competing effects of pore size reduction between convex borders of aggregates and gain in porosity at the nonconvex borders that are captured in a simple model fitting nicely the simulation data both in the isotropic and sheared packings. On the other hand, the internal angle of friction increases linearly with nonconvexity and saturates to a value independent of nonconvexity. We show that fabric anisotropy, force anisotropy, and friction mobilization, all enhanced by multiple contacts between aggregates, govern the observed increase of shear strength and its saturation with increasing nonconvexity. The main effect of interlocking is to dislocate frictional dissipation from the locked double and triple contacts between aggregates to the simple contacts between clusters of aggregates. This self-organization of particle motions allows the packing to keep a constant shear strength at high nonconvexity.
\end{abstract}

DOI: 10.1103/PhysRevE.84.041302

PACS number(s): 45.70.-n, 61.43.-j, 83.80.Fg

\section{INTRODUCTION}

Most of recent research work on granular physics has been concerned with the emerging behavior of a large number of basically monodisperse spherical particles [1,2]. The fact that a minimalistic model of granular matter reveals a complex generic rheology implies that the granular physics can be founded on a robust common denominator in spite of the wide variety of granular materials with substantial differences in their properties [3]. However, while essential for federative research on granular matter, this fact tends at the same time to eclipse the complexity arising from specific features of particles and their interactions.

Chief among those features is particle shape. The particles occur with various degrees of sphericity, elongation, angularity, facetedness, and convexity. In analogy to friction, particle shape affects the flow and equilibrium states of granular materials. For example, faceted particles present a higher shear strength than spherical particles [4-6], and packings of elongated particles may approach unusually high or low packing fractions [7-10]. Obviously, given the wide range of shapes and their degree of regularity in conjunction with particle size distribution, the mechanisms by which particle shape comes into play are diverse, and they hardly begin to be understood on quantitative grounds $[11,12]$. In this respect, the sphere packing model may be considered as reference material for the analysis of granular media with more general particle shapes deviating from a spherical or circular shape $[6,13]$.

Among various shape characteristics, nonconvexity is of special interest as it conveys the possibility of interlocking between particles. For example, in sintered powders such as $\mathrm{UO}_{2}$ pellets used as nuclear fuel, the particles at the micrometric scale are solid nonconvex aggregates composed of convex crystallites of nearly round shape [14]. The nonconvex feature of the aggregates varies from superficial roughness of their surface to deep concavity, allowing equally for large pores between aggregates and interlocked structures that vary with the degree of compaction.
We consider in this paper a two-dimensional (2D) model of nonconvex particles in which the degree of nonconvexity can be varied and that can easily be simulated by discrete element methods. These model particles are rigid aggregates of three overlapping disks with a three-fold rotational symmetry (see Fig. 1). Their nonconvexity can be tuned by adjusting the overlap, the range of shapes varying thus from disk, for a full overlap of the three disks, to a trimer of three tangent disks.

We perform quasistatic shear deformations of large packings of those model aggregates by means of the contact dynamics method [15,16]. We are interested both in the space-filling properties and shear strength as a function of nonconvexity. The shape may affect the behavior through various mechanisms such as the structure (packing texture and compactness) induced by shape nonconvexity, hindrance of particle rotations due to interlocking, enhanced mobilization of friction, and multiple contacts between aggregates with an effect similar to that of face-to-face contacts between faceted particles $[6,10,13]$. We analyze various internal variables in the initially isotropic packings prepared by isotropic compaction and in the sheared packings in order to determine the prevailing mechanisms with increasing nonconvexity of the aggregates.

In the following, we first introduce in Sec. II the technical details of the simulations, procedures of sample preparation and relevant mechanical observables, which are essential for the interpretation of the results. Then, in Sec. III, we present the evolution of shear stress and packing fraction with shear strain and at an increasing level of nonconvexity. As we shall see, the shape dependence is rather complex and characterized by a saturation of shear strength at high nonconvexity and unmonotonic evolution of the packing fraction. Sections IV, V, VI, and VII are devoted to the analysis of contact network topology, force distributions, friction mobilization, and forcecontact anisotropy. In Sec. VIII, we introduce a simple model of packing fraction as a function of nonconvexity that nicely fits our simulation data. We conclude with a discussion of the most salient results of this work and its possible extension to three dimensions and more general particle shapes. 


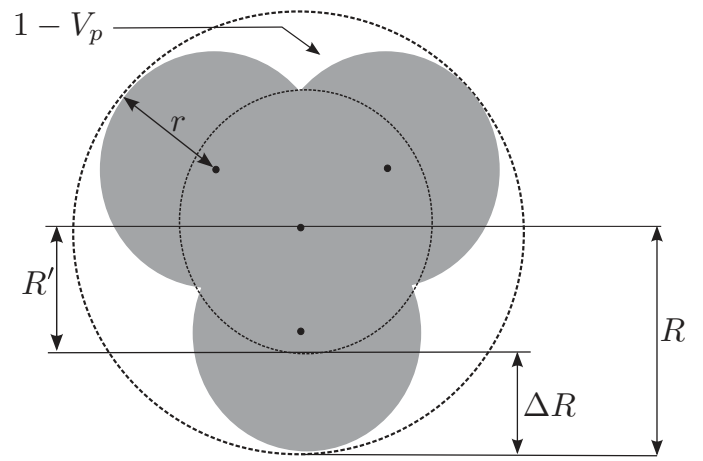

FIG. 1. Geometry of a regular aggregate.

\section{SYSTEM DESCRIPTION}

\section{A. Particle shape parameter}

Figure 1 shows a regular aggregate of threefold rotational symmetry composed of three overlapping disks of the same radius $r$. The shape of an aggregate can be characterized by considering the radius $R$ of the circumscribing circle as compared to the radius $R^{\prime}$ of the inscribed circle. The difference $\Delta R=R-R^{\prime}$ represents the concavity of the aggregate, that is, the inward deviation from the surface of the circumscribing circle. Hence, we define the nonconvexity $\eta$ of an aggregate by the ratio

$$
\eta=\frac{\Delta R}{R} .
$$

An alternative parameter of nonconvexity for aggregates is the ratio $\lambda \equiv \ell / 2 r$, where $\ell$ is the distance between disk centers. The parameter $\eta$ can be expressed as a function of $\lambda$ :

$$
\eta=\frac{3+\sqrt{3} \lambda-3 \sqrt{1-\lambda^{2}}}{3+2 \sqrt{3} \lambda} .
$$

It varies from $\eta=0$, corresponding to a disk $(\lambda=0)$, to $\eta \simeq$ 0.73 , corresponding to a trimer of three disks of vanishing overlap $(\lambda=1)$. The parameter $\eta$ can also be related to the notion of "roundness" expressed in geology as the ratio of the average radius of curvature of the edges or corners to that of the maximum inscribed sphere $[17,18]$. With our notations, the roundness of the aggregates is simply $r /[(1-\eta) R]$, which decreases from 1.72 for $\lambda=0$ to $\simeq 0.367$ for $\lambda=1$.

\section{B. Numerical method}

The simulations were carried out by the contact dynamics method $[2,15,19,20]$. Newton's equations of motion for all rigid-body degrees of freedom of all particles are integrated by taking into account contact reaction forces between particles, as well as bulk forces such as gravity. In contrast to the molecular dynamics method [21-24], where an explicit scheme is used for solving the equations of motion by introducing stiff repulsive potential and viscous damping between particles, the contact dynamics method is based on an implicit scheme involving an iterative Gauss-Seidel algorithm yielding simultaneously the contact forces and particle displacements at the end of each time step. This iterative process is defined such as to satisfy the kinematic constraints related to mutual exclusions of particles and the Coulomb friction law. For this reason, the contact dynamics method is unconditionally stable and does not require elastic repulsive potential between particles. This allows for much larger time steps than in molecular dynamics, in particular in the limit of highly stiff particles or very low confining stresses.

It should also be noticed that the solution is not generally unique when dealing with perfectly rigid particles. However, the variability of the solution is small and often in the limit of numerical precision as the kinematic constraints and disorder prevail in granular dynamics. In practice, the evolution of the system can be made almost deterministic by initializing the iterative process at each step by the contact forces at the end of the preceding step [19]. This point has been investigated by comparison with molecular dynamics simulations and experiments [25-29].

When treating complex-shaped particles such as aggregates, the same iterative process can be applied although several contact points may occur between two particles (see Fig. 7). The contacts, including those occurring between two neighboring particles, are treated as independent unilateral constraints [30]. The reaction force between two particles is the result of all forces acting at individual contacts.

\section{Sample preparation}

Eight samples of 5000 aggregates were prepared for eight different values of $\eta \in[0,0.7]$. To avoid long-range ordering, a size polydispersity was introduced by taking $R$ in the range [ $R_{\min }, R_{\max }$ ] with $R_{\max }=3 R_{\min }$ and a uniform distribution of particle volumes $\left(\propto R^{-2}\right)$, which leads to a high packing fraction $\rho$ [31-34]. It is worth mentioning that a lower level of size polydispersity might be sufficient for topological disorder (disorder in the connectivity of the particles and force transmission) but does not ensure the metric disorder of the contact network.

A dense packing composed of disks $(\eta=0)$ was first constructed by means of random deposition inside a rectangular box of dimensions $l \times h$. For other values of $\eta$, the same packing was used with each disk serving as the circumscribing circle. The particle was inscribed with the given value of $\eta$ and random orientation inside the disk. This geometrical step was followed by isotropic compaction of the packings by the contact dynamics method inside a rectangular frame. The gravity $g$ and friction coefficients $\mu$ and $\mu_{w}$ between the particles and with the walls, respectively, were set to zero during compaction to ensure packing homogeneity. The samples prepared by this procedure are both isotropic and isostatic, as we see in Sec. IV. Figure 2 displays snapshots of four packings for increasing value of $\eta$ at the end of isotropic compaction.

These isotropic samples were then sheared biaxially by applying a slow downward velocity $\dot{y}$ on the top wall with a constant confining stress acting on the lateral walls. During shear, the friction coefficient was set to 0.5 between particles and to 0 with the walls. The zero friction with the walls prevents from stress gradients as those that lead to the Janssen effect [35]. The vertical shear rate $\dot{y} / y$ is low enough to ensure a 

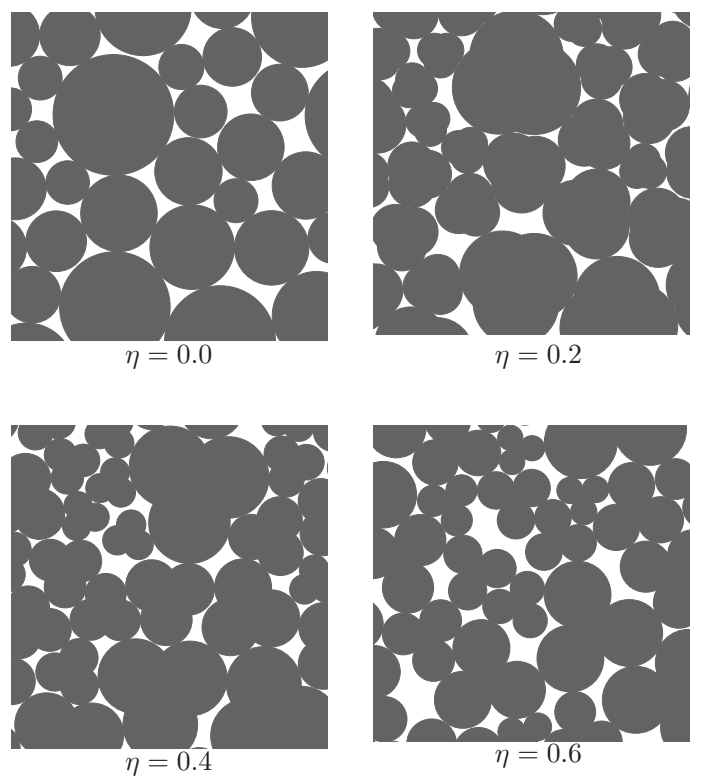

FIG. 2. Snapshots of the numerical samples at the end of isotropic compaction with zero friction between particles for different values of shape parameter $\eta$.

quasistatic state during shear by reducing the inertia parameter $I$ given by (in 2D) [36]:

$$
I=\frac{\dot{y}}{y} \sqrt{\frac{m}{p}},
$$

where $m$ is the particle mass and $p$ is the mean pressure by unit length. In our simulations, $I$ was below $10^{-3}$. Each sample is sheared until a residual state is reached with a nearly constant packing fraction. ${ }^{1}$

\section{Stress and strain variables}

In numerical simulations, the stress tensor can be evaluated from the contact forces and the geometrical configuration of a packing [37]. An interesting approach for the estimation of the stress tensor was introduced by Moreau [38]. It is based on the virtual power formalism and the notion of "internal moment" $\boldsymbol{M}^{i}$ defined for each particle $i$ in the packing as

$$
M_{\iota \delta}^{i}=\sum_{c \in i} f_{\iota}^{c} r_{\delta}^{c},
$$

where $f_{L}^{c}$ is the $\iota$ component of the force exerted on particle $i$ at the contact $c, r_{\delta}^{c}$ is the $\delta$ component of the position vector of the same contact $\mathrm{c}$, and the summation runs over all contact neighbors of particle $i$ (noted briefly by $c \in i$ ).

It can be shown that the internal moment of a collection of rigid particles is the sum of the internal moments of individual particles, and the stress tensor $\sigma$ is simply the density of internal moment [38]:

$$
\boldsymbol{\sigma}=\frac{1}{V} \sum_{i \in V} \boldsymbol{M}^{i}=\frac{1}{V} \sum_{c \in V} \boldsymbol{f}_{\imath}^{c} \boldsymbol{l}_{\delta}^{c}
$$

\footnotetext{
${ }^{1}$ Animation videos of these simulations are available at [www.cgpgateway/ref007].
}

where $V$ is the volume of the packing or a domain inside the packing and $\ell^{c}$ is the branch vector joining the centers of the two touching particles at the contact $c$. In view of the above definition, which can be shown to be equivalent to other expressions of the stress tensor $[2,39]$, the internal moment tensor in a granular packing at equilibrium underlies the Cauchy stress tensor which is a notion of continuum mechanics.

In biaxial compression, the major principal stress direction coincides with the compression axis and the principal stress values are $\sigma_{1}$ along the compression axis and $\sigma_{2}$ along the extension axis. The mean stress $p$ and the deviatoric stress $q$ are defined by

$$
\begin{aligned}
& p=\frac{1}{2}\left(\sigma_{1}+\sigma_{2}\right), \\
& q=\frac{1}{2}\left(\sigma_{1}-\sigma_{2}\right) .
\end{aligned}
$$

For a system of rigid particles, the stress state is fully defined by the dimensionless ratio $q / p$.

The strain variables are defined from the deformations of the simulation box. Let $h_{0}$ and $l_{0}$ be the initial height and width of the simulation box. The compression being in the vertical direction, the cumulative principal strains $\varepsilon_{1}$ and $\varepsilon_{2}$ are given by

$$
\varepsilon_{1}=\int_{h_{0}}^{h} \frac{d h^{\prime}}{h^{\prime}}=\ln \left(1+\frac{\Delta h}{h_{0}}\right),
$$

where $h$ is the current height and $\Delta h=h_{0}-h$ is the total downward displacement, and

$$
\varepsilon_{2}=\int_{l_{0}}^{l} \frac{d l^{\prime}}{l^{\prime}}=\ln \left(1+\frac{\Delta l}{l_{0}}\right),
$$

where $l$ is the current box width and $\Delta l=l-l_{0}$ is the total change of the box width.

The cumulative shear strain is defined by

$$
\varepsilon_{q} \equiv \varepsilon_{1}-\varepsilon_{2},
$$

whereas the cumulative volumetric strain $\varepsilon_{p}$ is given by

$$
\varepsilon_{p}=\varepsilon_{1}+\varepsilon_{2}=\int_{V_{0}}^{V} \frac{d V^{\prime}}{V^{\prime}}=\ln \left(1+\frac{\Delta \rho}{\rho}\right),
$$

where $V_{0}=l_{0} h_{0}$ is the initial volume and $\Delta \rho=\rho-\rho_{0}$ is the cumulative change of packing fraction.

\section{MACROSCOPIC BEHAVIOR}

We are interested in this section in the effective quasistatic rheology of the packings of aggregates in terms of shear strength and evolution of packing fraction under monotonic loading for increasing values of the nonconvexity parameter $\eta$.

\section{A. Shear strength}

Figure 3 displays the normalized shear stress $q / p$ as a function of the cumulative shear strain $\varepsilon_{q}$ for different values of the nonconvexity parameter $\eta$. As a consequence of sample preparation by isotropic compaction, $q / p$ is vanishingly small in the initial isotropic state $\left(\varepsilon_{q}=0\right)$. It jumps to a finite value as soon as the shear is applied and then relaxes to a 


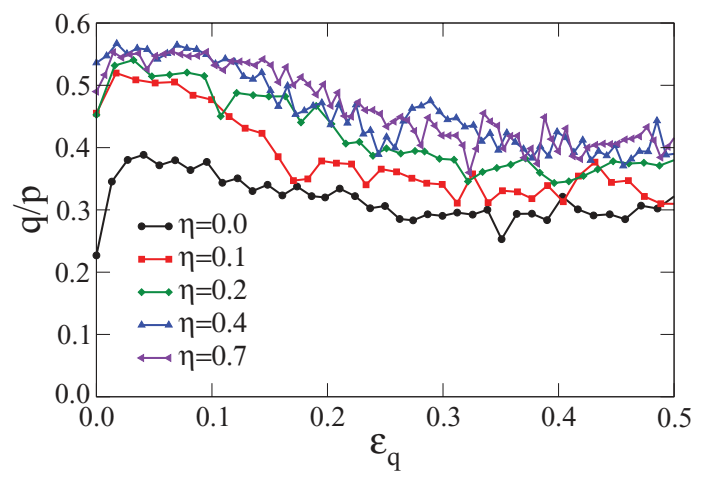

FIG. 3. (Color online) Normalized shear stress as a function of the cumulative shear strain for different values of the shape parameter $\eta$.

constant residual shear stress up to fluctuations of rather small amplitude. The initial jump to the peak stress reflects the fact that the samples have a high packing fraction in the initial state, and the particles are assumed to be perfectly rigid and simulated as such by the contact dynamics method [15]. We see that both the peak stress and the residual stress increase with $\eta$. The normalized residual stress $q^{*} / p$ is independent of initial state and it represents the intrinsic shear strength of the material that often is described by the internal angle of friction $\varphi^{*}$ defined in $2 \mathrm{D}$ by

$$
\sin \varphi^{*}=\frac{q^{*}}{p} .
$$

Figure 4 shows $\sin \varphi^{*}$ as a function of $\eta$. The error bars represent the standard deviation computed from the fluctuations around the mean in the residual state as observed in Fig. 3. It can be seen that, up to the fluctuations, $\sin \varphi^{*}$ increases linearly from $\simeq 0.3$ to $\simeq 0.43$ at $\eta=0.5$ and beyond, where it stays independent of $\eta$. This increase of shear strength reflects the effect of nonconvexity on the internal structure, as we see in Sec. VII. However, it is surprising that this effect is apparently absent for $\eta>0.4$. In a recent work by Azema and Radjai [10] granular packings of elongated particles were investigated and a parameter similar to $\eta$ was used. They found a similar trend of increasing shear strength with $\eta$ with nearly the same values but, in contrast to our nonconvex particles, no

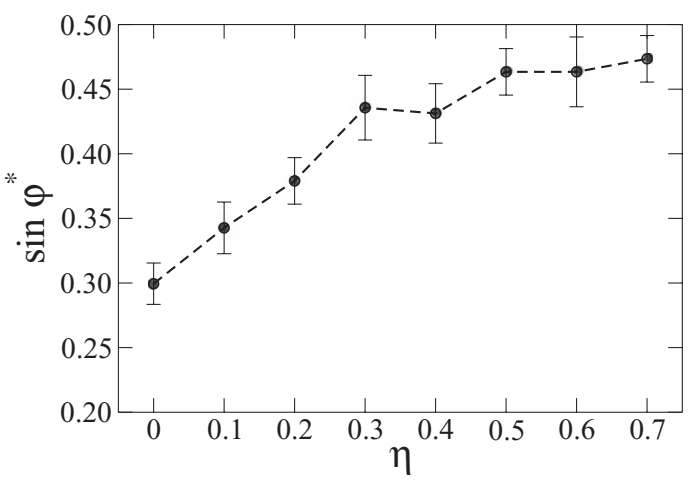

FIG. 4. Internal angle of friction $\varphi^{*}$ as a function of $\eta$. Error bars represent the standard deviation in the residual state.

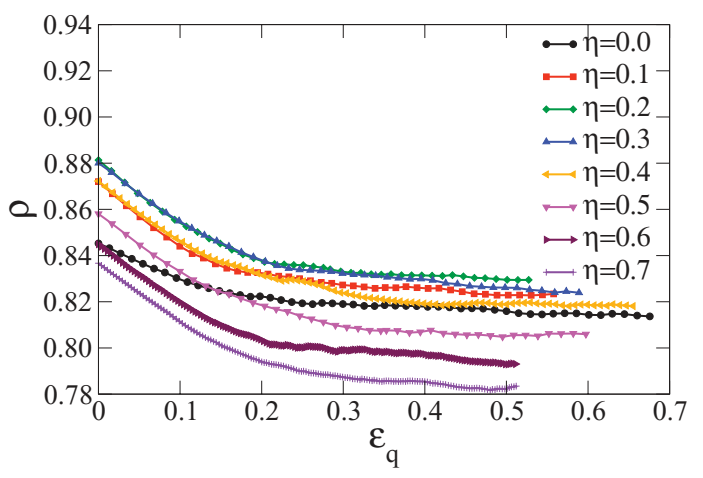

FIG. 5. (Color online) Evolution of packing fraction $\rho$ with the cumulative shear strain $\varepsilon_{q}$ for different values of $\eta$.

saturation of shear strength was observed. We come back to this point below when analyzing the microstructure.

\section{B. Packing fraction}

In Fig. 5, the evolution of packing fraction $\rho$ is shown as a function of $\varepsilon_{q}$ for different values of $\eta$. All samples dilate during shear and $\rho$ declines from its value $\rho^{\text {iso }}$ in the initial isotropic state down to a constant value $\rho^{*}$ in the residual state. The samples dilate almost homogeneously at low shear strains $(\leqslant 0.2)$ and thus $\rho$ decreases rapidly. At larger strains, dilation is localized within shear bands appearing throughout the system. As the shear bands develop at different locations inside the system, a nearly homogeneous density $\rho^{*}$ is reached practically at $\varepsilon_{q}=0.5$. For our rigid particles the residual packing fraction $\rho^{*}$ is independent of the confining pressure and, as $q^{*} / p$, it should be considered as an intrinsic property of the material, that is, reflecting basically the particle shape and size distribution as well as the friction coefficient between particles.

Figure 6 displays $\rho$ as a function of $\eta$ at different instants of $\varepsilon_{q}$. Remarkably, $\rho$ first grows from its value for disks $(\eta=0)$ toward a maximum at $\eta \simeq 0.2$ and then declines at higher values of $\eta$. The peak value of packing fraction $\rho^{\text {iso }}$ in the isotropic state is as high as 0.88 . In the residual state, the packing fraction $\rho^{*}$ takes as low value as 0.78 at $\eta=0.7$. A similar unmonotonic behavior of packing fraction has been previously observed for granular packings of elongated particles such as ellipses, ellipsoidal particles, spherocylinders,

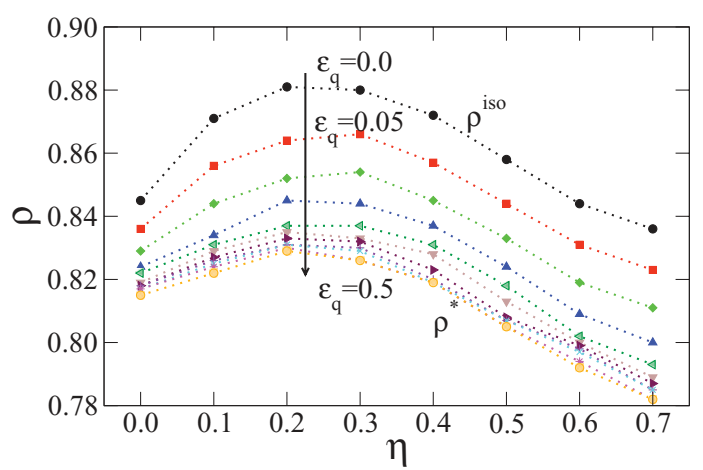

FIG. 6. (Color online) Packing fraction $\rho$ as a function of $\eta$ at different levels of cumulative shear strain $\varepsilon_{q}$. 
and rounded-cap rectangles $[7,8,10,40]$. The falloff of the packing fraction at higher aspect ratios is attributed to the increase of excluded volumes, that is, the largest pore volume that can not be filled by a particle. Obviously, a similar effect can be advocated for our nonconvex particles which can form an increasingly tortuous and large pore space as the nonconvexity increases. However, the initial increase of $\rho_{0}$ as $\eta$ varies from 0 to 0.2 indicates that this effect does not prevail at low $\eta$ corresponding to small deviations from a reference circular shape. As we see below, this effect and the subsequent decrease of $\rho$ result from the size variation of different classes of pores. We also guess that there is a link between the decrease of $\rho$ and the saturation of $\varphi^{*}$ at large values of $\eta$. Indeed, it seems plausible that the increase of free volume prevents from further increase of interlocking although the particle nonconvexity becomes higher, so that the shear strength remains constant. This picture emerges also from the analysis of microstructure (Sec. IV).

\section{CONTACT NETWORK TOPOLOGY}

In this section, we analyze the microstructure of our simulated packings of aggregates as a function of particle shape nonconvexity. The relevant microstructural variables in a granular material are linked with the contact network, which is the backbone of stress transmission in quasistatic equilibrium [3]. The principal effect of shape nonconvexity is to allow for multiple contacts between aggregates as shown in Fig. 7. Four different types of contact can occur between two aggregates as shown in Fig. 7: (1) simple contact (S), (2) double-simple (DS) contact, defined as two simple contacts between two pairs of disks belonging to the aggregates, (3) double (D) contact, defined as two contacts between one disk of one aggregate with two disks of the other aggregate, and (4) triple (T) contact, defined as the combination of one simple and one double contact.

Given multiple contacts between aggregates, we distinguish between the coordination number $Z$, defined as the mean number of contact neighbors per particle, and the connectivity number $Z_{c}$, defined as the mean number of contacts per particle. For convex particles, we have $Z=Z_{c}$. The contact network of nonconvex particles can thus be characterized by $Z$ and the ratio $K=Z_{c} / Z$. Let $K_{S}, K_{D S}, K_{D}$, and $K_{T}$ be the proportions of simple, double simple, double, and triple contacts. It is easy to see that

$$
K=\frac{Z_{C}}{Z}=K_{S}+2\left(K_{D S}+K_{D}\right)+3 K_{T} .
$$

Figure 8 shows the coordination number in the initial (isotropic) and residual states as a function of $\eta$. Quite interestingly, $Z^{*} \simeq 3$ is nearly independent of $\eta$, whereas
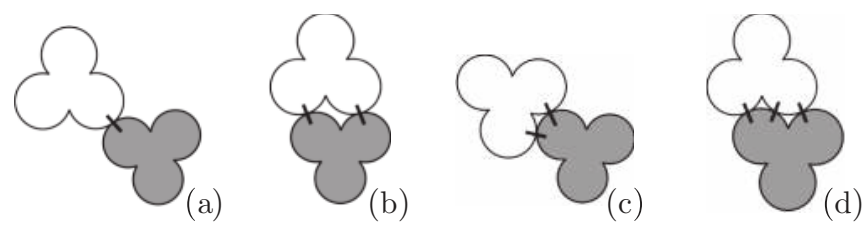

FIG. 7. Four different contact configurations between two aggregates: (a) simple, (b) double simple, (c) double, and (d) triple.

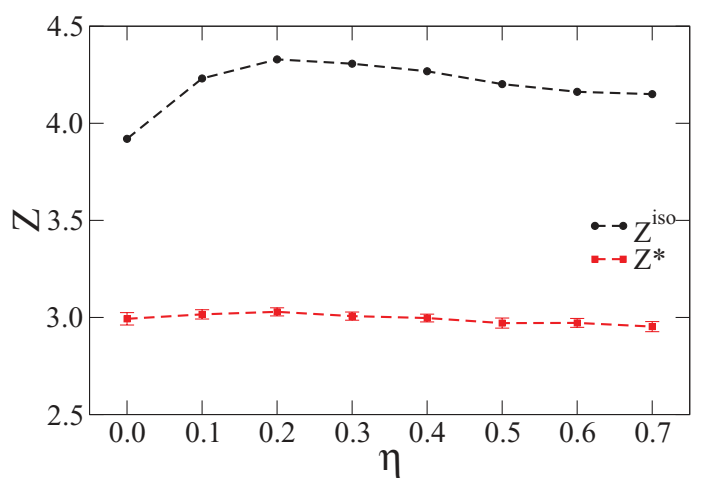

FIG. 8. (Color online) Coordination number $Z$ in the initial isotropic state (labeled by "iso") and in the residual state (labeled by $\star$ ) as a function of nonconvexity $\eta$. The error bars of $Z^{*}$ indicate the standard deviation of the data in the residual state.

$Z^{\text {iso }}$ follows the same trend as $\rho^{\text {iso }}$ (see Fig. 6). The higher value of $Z^{\text {iso }}$ is a consequence of isotropic compaction with zero friction. Its value then declines to $Z^{*}$ as a result of dilatancy by shearing with $\mu=0.5$. The rather low value $Z^{*} \simeq 3$ implies that many aggregates have only three contact neighbors irrespective of nonconvexity.

The connectivity number $Z_{c}$ is displayed in Fig. 9 as a function of $\eta$. Its value in the isotropic state jumps from $Z_{c}^{\text {iso }}=4$ at $\eta=0$ to $Z_{c}^{\text {iso }} \simeq 6$ at $\eta=0.1$. Both values are compatible with the isostatic nature of our packings prepared with a zero friction coefficient [41-43]. Indeed, frictionless circular particles $(\eta=0)$ are characterized by two degrees of freedom (rotations being immaterial) and thus the isostatic condition implies two independent constraints (normal forces) which amounts to a connectivity number of 4 . For slightest deviation from circular shape the rotation becomes material and a similar counting argument leads to a connectivity number of 6 . In contrast, for frictional aggregates in the residual state, $Z_{c}^{*}$ increases from 3 to $\simeq 3.8$ at $\eta=0.7$. The effect of increasing nonconvexity is therefore expressed by an increasing number of multiple contacts with the same average number of neighboring aggregates $(Z \simeq 3$ ). It is remarkable that $Z_{c}^{*}$ does not follow the trend of the packing fraction (Fig. 25) as $\eta$ is increased, so that at high nonconvexity the

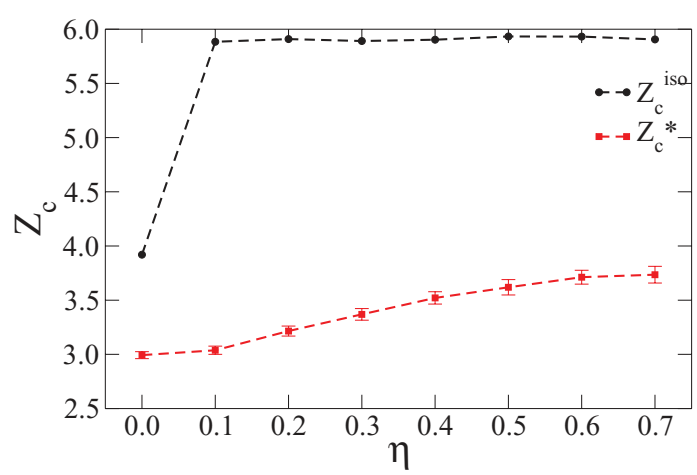

FIG. 9. (Color online) Connectivity number $Z_{c}$ in the initial isotropic state (labeled by "iso") and in the residual state (labeled by $\star$ ) as a function of nonconvexity $\eta$. The error bars of $Z_{c}^{*}$ indicate the standard deviation of the data in the residual state. 


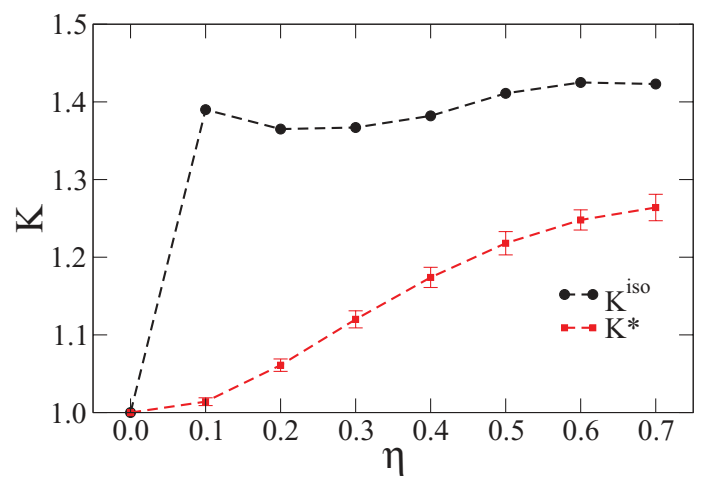

FIG. 10. (Color online) The ratio $K$ of connectivity number to coordination number in the initial isotropic state (labeled by "iso" or black line) and in the residual state (labeled by $\star$ or red line) as a function of nonconvexity $\eta$. The error bars of $K^{*}$ indicate the standard deviation of the data in the residual state.

packings of aggregates are less compact but more tightly connected. The ratio $K$ reflects this behavior as shown in Fig. 10. The isostatic packings are characterized by a ratio $K^{\text {iso }} \simeq 1.4$ for $\eta>0$, whereas the sheared packings in the residual state have a ratio $K^{*}$ increasing smoothly from 1 to 1.26 as $\eta$ varies from 0 to 0.7 .

Figure 11(a) displays the proportions of different contact types as a function of $\eta$ in the isostatic state. We observe that, like $Z^{\text {iso }}$ and $Z_{c}^{\text {iso }}$, the proportions of different types of connection between aggregates are nearly independent of $\eta$ in the isostatic state. The simple contacts represent the highest proportion $(\simeq 0.63)$, whereas the triple and double-simple contacts have the lowest proportions $(<0.1)$. The double

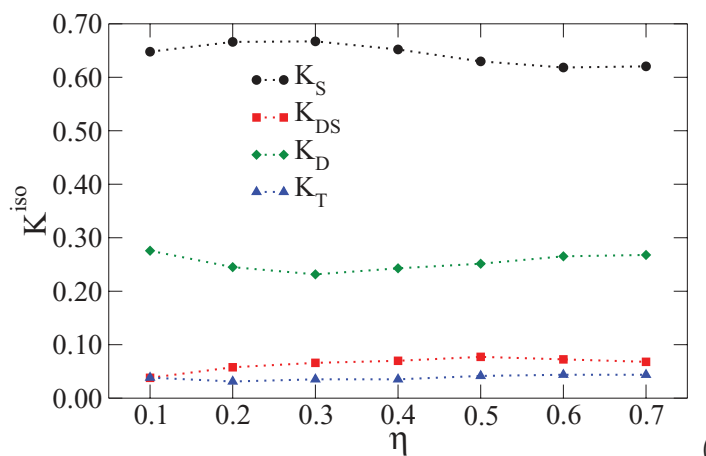

(a)

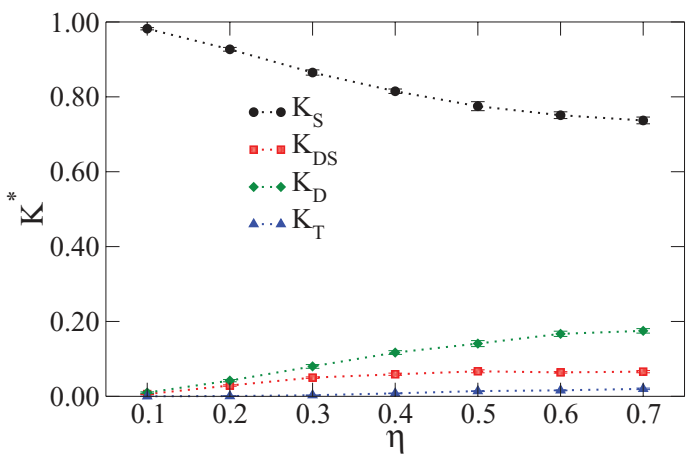

FIG. 11. (Color online) Proportions of simple, double, doublesimple, and triple contacts as a function of $\eta$ in the isotropic (a) and residual (b) states. contacts are represented by an intermediate proportion about 0.27 . The fractions of contact types in the residual state are displayed in Fig. 11(b). The proportion of simple contacts declines as $\eta$ is increased but its value remains above that in the isotropic state at the expense of double contacts, which are fewer in number.

One observes also a drastic loss of double contacts in the residual state compared to the initial isotropic state for all values of $\eta$. In contrast, the proportion of double-simple and triple contacts are nearly the same. In fact, the isostatic state corresponds to the unique minimum of the total potential energy $p V$. This state is achieved by an enhanced number of double and triple contacts, which tend to decrease the volume. The situation is different in the residual state, which is governed by shear-induced dilatation of the material and where the particles explore constantly metastable states. In this state, the double and triple contacts are no more required for the equilibrium and stability of the packing. However, as we see below, the role of interlocking due to a weak increase of the number of double and triple contacts is to reduce energy dissipation by allowing for clustered rigid rotations of the paricles (see Sec. VI).

The description of the microstructure in terms of the average coordination and connectivity between the aggregates, provides us with a basic picture of the effect of shape nonconvexity. The isotropic states prepared by compaction with zero friction between aggregates are interesting but rather special isostatic states that exhibit practically no dependence on particle shape. The residual state, on the contrary, reveals a clear shape dependence, marked out by increasing connectivity though with a nearly constant coordination number as the shape nonconvexity increases. In contrast to the unmonotonic evolution of the packing fraction, the increasing connectivity of the aggregates is obviously correlated with the increase of shear strength. In the following, we analyze force transmission and higher-order descriptors of the microstructure in order to clarify the nature of this correlation.

\section{FORCE DISTRIBUTIONS}

The distribution of contact forces in granular media reflects the inhomogeneity of the contact network. For our packings of aggregates one expects an invariant distribution in the isotropic state since the connectivity remains unchanged as nonconvexity is increased. This is what we observe in Figs. 12(a) and 12(b), where the probability density functions (PDFs) of normal forces $f_{n}$ are displayed. The PDF for $\eta \neq 0$ is practically the same as for disks $(\eta=0)$ with a roughly exponential falloff of large forces, a peak slightly below the mean force and a nonvanishing PDF as the force tends to zero [44-52]. The normal force PDFs in the residual state are shown in Figs. 12(c) and 12(d). Here the distribution is increasingly broader as nonconvexity is increased. We observe an increasing number of weak forces and increasingly stronger forces. The exponential character $\left[P\left(f_{n}\right) \propto e^{-\alpha_{n} f_{n} /\left\langle f_{n}\right\rangle}\right]$ of the distribution in the range of strong forces is more pronounced compared to that in the isostatic state as the extent of strong forces increases with $\eta$. It is characterized by the exponent $\alpha_{n}$, which declines from $\simeq 1.71$ to 1 as $\eta$ increases from 0 to 0.7 . The distribution in the range of weak forces may be 


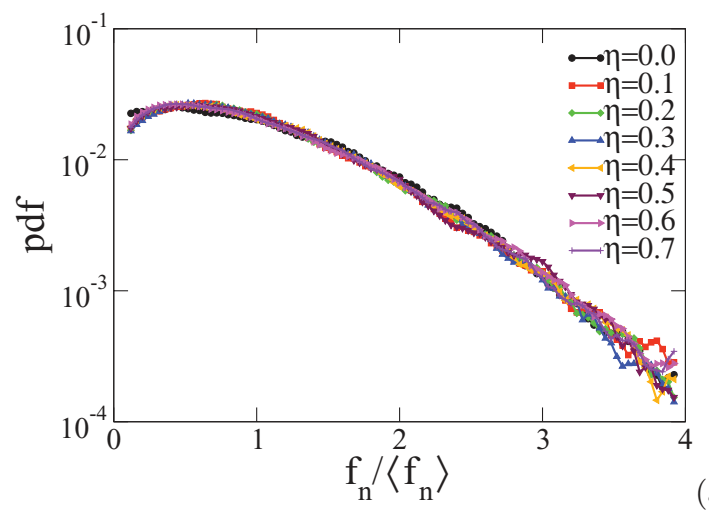

(a)
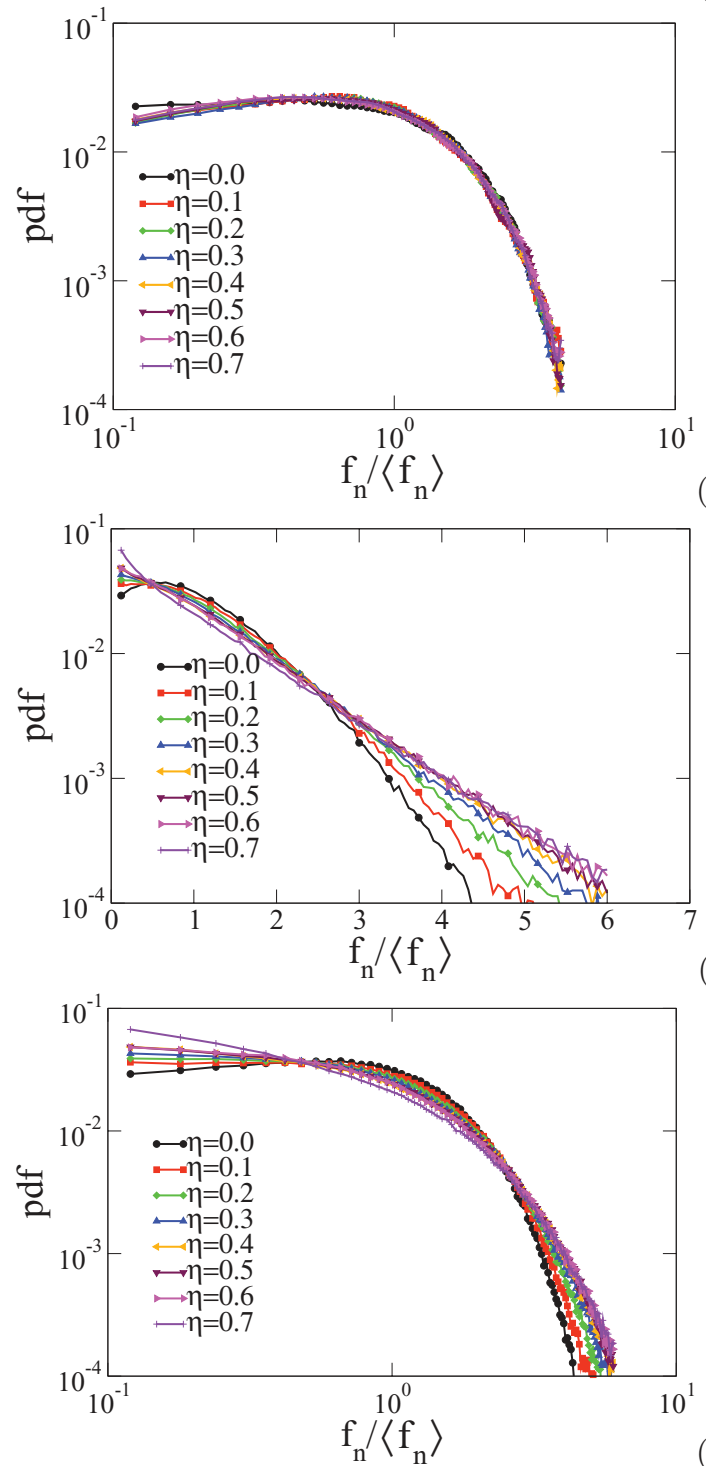

FIG. 12. (Color online) Probability distribution function of normal forces for different levels of shape nonconvexity $\eta$ in the isotropic (a),(b) and residual (c),(d) states in a log-linear and log-log scales. The forces are normalized by the mean normal force.

approximated by a power law $\left[P\left(f_{n}\right) \propto f_{n} /\left\langle f_{n}\right\rangle^{-\beta_{n}}\right]$, as seen on the $\log -\log$ scale with the exponent $\beta_{n}$ increasing from $\simeq-0.16$ for $\eta=0$ to $\simeq 0.46$ for $\eta=0.7$. This means that the packings of more nonconvex aggregates, though more closely connected, are more inhomogeneous. This discrepancy may be
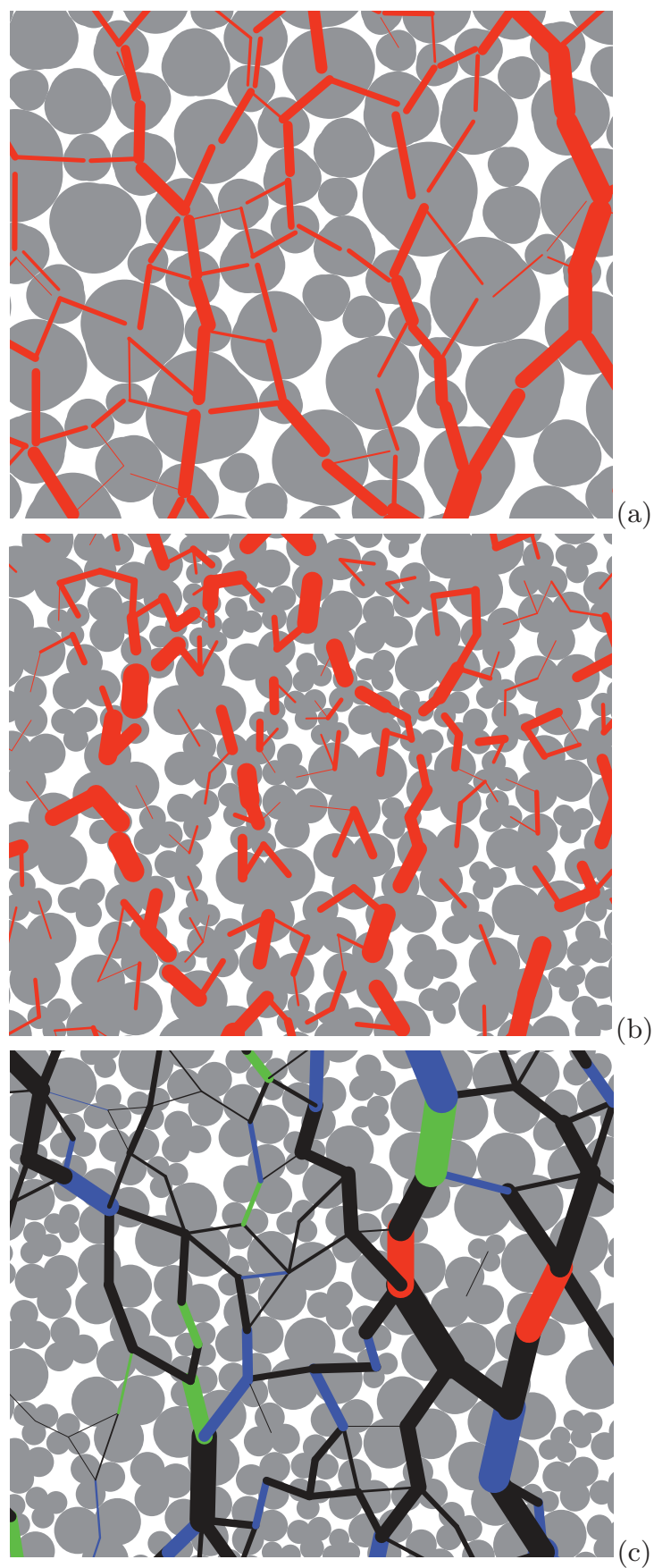

FIG. 13. (Color online) Snapshots of normal force chains for $\eta=0.1$ (a), $\eta=0.5$ (b), and radial force chain for $\eta=0.5$ (c) taken in the residual state. Line thickness is proportional to force intensity. In (c) the black, dark gray, medium gray, and light gray lines are, respectively, radial forces for simple, double, double-simple, and triple contacts.

attributed to enhanced disorder due to the increasing number of multiple contacts between aggregates. Figures 13(a) and 13(b) show two snapshots of normal forces for $\eta=0.1$ and $\eta=0.5$ in the residual state. We observe double contacts with double force lines as well as enhanced force branching for $\eta=0.5$. The visual aspect of the force chains is consistent with a higher inhomogeneity for $\eta=0.5$. 
In order to highlight the role of multiple contacts in force transmission, we consider the reaction forces between aggregates. The reaction force $\boldsymbol{F}$ between two aggregates is the resultant of point forces acting at their contacts. It can be projected on the branch vector connecting the centers of the two aggregates with unit vector $\boldsymbol{n}^{\prime}$. In this way, the complex network of contacts can be replaced by the simpler network of branch vectors and carrying the radial force $F \equiv \boldsymbol{F} \cdot \mathbf{n}^{\prime}$ between aggregates [6,13]. This network of neighbors can be compared to that of convex particles and among systems with different levels of shape nonconvexity. One example is displayed in Fig. 13(c), which represents the radial forces $F$ extracted from the contact force. The radial force chains observed in this figure have nearly the same feature as those generally observed between convex particles.

We differentiate the average radial forces $F_{S}, F_{D}, F_{D S}$, and $F_{T}$ of simple, double, double-simple, and triple contacts, respectively. Figures 14(a) and 14(b) display the the radial forces of different contact types normalized by the average normal force $\left\langle f_{n}\right\rangle$ as a function of $\eta$ in the isotropic and residual states. One remarkable feature is that these averages by contact type are practically independent of $\eta$ in the isotropic state. This means that the average reaction force of each contact type varies as the average normal force. $F_{S}^{\text {iso }}$ remains close to 1 (of the order of the average normal force), whereas $F_{D}^{\text {iso }}$ and $F_{D S}^{\text {iso }}$ are slightly below two. Since each double or double-simple contact involves two contacts, this observation also means that the two contact forces belonging to a double contact between a pair of aggregates are almost statistically independent forces. In contrast, we observe that $F_{T}^{\text {iso }}<2.5$, implying that the three

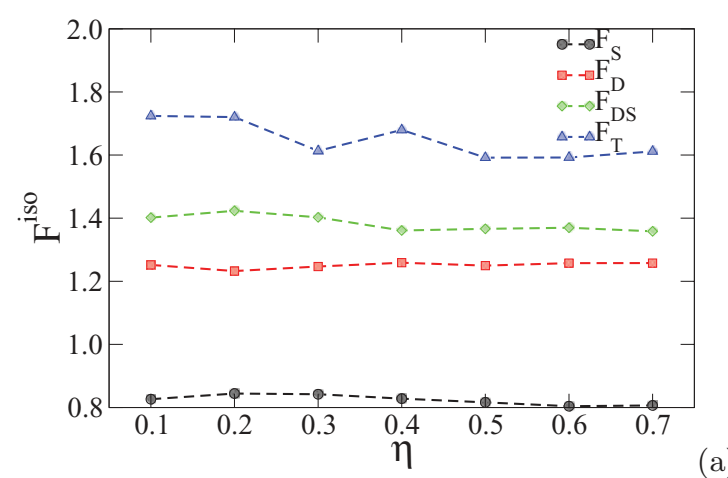

(a)

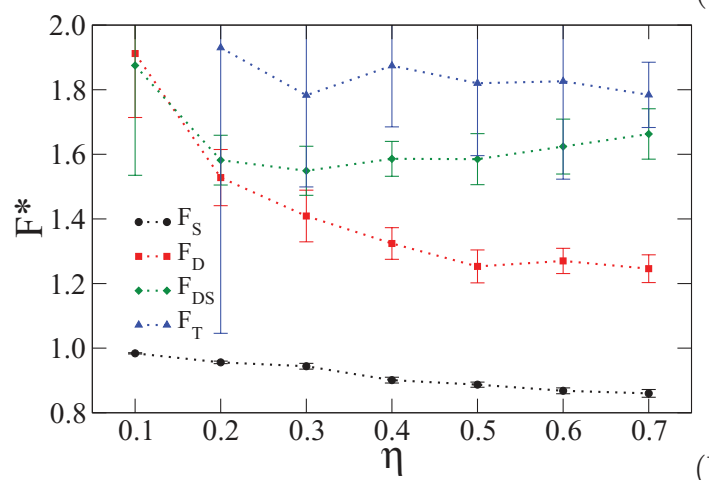

FIG. 14. (Color online) Average forces by contact type normalized by the average normal force as a function of $\eta$ in the isotropic (a) and residual (b) states. forces belonging to a triple contact are generally correlated since one would have $F_{T}^{\text {iso }}=3$ otherwise.

In the residual state, $F_{S}^{*}$ declines slightly from 1 with increasing $\eta$ and $F_{T}^{*}$ remains unchanged and close to 1.8 up to error bars due to the weak number of triple contacts at low values of $\eta . F_{D}^{*}$ falls off to very low values $(\simeq 1.2)$, a sign of very high correlation between the two point forces at the two contact points between one disk of one aggregate and two disks of another aggregate (we would have $F_{D}^{*}=2$ without correlation). This correlation may be attributed to the increasing level of kinematic constraint or interlocking as nonconvexity increases (see Sec. VI). In contrast, $F_{D S}^{*}$ varies in the range $[1.6,1.9]$.

It is important to note that multiple contacts, though far less in number than simple contacts (Fig. 11), carry the largest forces and represent the strong radial force chains in Fig. 13(c). Their respective weights in force transmission are, however, given by $K_{D} F_{D}, K_{D S} F_{D S}$, and $K_{T} F_{T}$, which are below $K_{S} F_{S}$, but grow as $K_{D}, K_{D S}$, and $K_{T}$ increase with $\eta$.

\section{FRICTION MOBILIZATION}

The degree of mobilization of friction forces is a basic state parameter in granular materials. The friction forces increase on average with shear stress, and sliding occurs only at a subset of contacts where the friction force $f_{t}$ equals in absolute value the friction coefficient $\mu$ times the normal force $f_{n}$. In the case of aggregates, an interesting issue is how far their nonconvex geometry affects friction mobilization and whether sliding is hindered by interlocking.

Let $\left(\mathbf{n}^{a}, \mathbf{t}^{a}\right)$ be a local frame of specified polarity attached to a contact $a$ of an aggregate, where $\mathbf{n}^{a}$ is the inward contact normal and $\mathbf{t}^{a}$ is the unit orthogonal vector. The components of the contact force $\mathbf{f}^{a}$ acting on the aggregate in this frame are the normal force $f_{n}^{a}$, which is positive, and the tangential force $f_{t}^{a}$, which can be either positive or negative within the Coulomb friction criterion $\left|f_{t}\right| \leqslant \mu f_{n}$. We have $\mathbf{f}^{a}=f_{n}^{a} \mathbf{n}^{a}+f_{t}^{a} \mathbf{t}^{a}$. The accelerations are assumed to be negligible in quasistatic deformation so that the forces and force moments acting on the aggregate are balanced:

$$
\begin{gathered}
\sum_{a} \mathbf{f}^{a}=0, \\
\sum_{a} h_{n}^{a} f_{t}^{a}=0,
\end{gathered}
$$

with $h_{n}^{a}=\mathbf{h}^{a} \cdot \mathbf{n}$, where $\mathbf{h}^{a}$ is the contact vector joining the center of inertia of the aggregate to the contact $a$.

Taking the average of the second equation in Eq. (15) over all aggregates and assuming that $h_{n}^{a}$ and $f_{t}^{a}$ are statistically independent, we get $\left\langle h_{n}\right\rangle\left\langle f_{t}\right\rangle=0$. Hence, we have $\left\langle f_{t}\right\rangle=0$ since $\left\langle h_{n}\right\rangle>0$. This means that, due to the balance of force moments, the average tangent force in the packing vanishes. This is what generally is observed in numerical simulations of granular materials. For this reason, the average tangential force does not reflect the mobilization of friction.

The friction mobilization can be defined from higher-order moments of tangential forces. At second order, the root mean square (rms) $\sqrt{f_{t}^{2}}$ of tangential forces provides a good measure of friction mobilization when normalized by the mean $\mu\left\langle f_{n}\right\rangle$. 
Alternatively, a friction mobilization index $I_{f}$ can be defined from the absolute values of friction forces:

$$
I_{f}=\left\langle\frac{\left|f_{t}\right|}{\mu f_{n}}\right\rangle,
$$

where the average is taken over all contacts. This definition is closer to the spirit of the Coulomb friction law as compared to the rms of friction forces.

A somewhat more elegant way of describing friction mobilization is to start with the probability density of the friction force $f_{t} \boldsymbol{t}$, which derives from the joint probability density $P\left(f_{t}, \boldsymbol{t}\right)$ of the friction force $f_{t}$ and its orientation $\boldsymbol{t}$. In $2 \mathrm{D}$, the vector $t=(-\sin \theta, \cos \theta)$ is parametrized by the orientation $\theta$ of the contact normal $\boldsymbol{n}$ and we have

$$
\left\langle f_{t}\right\rangle_{\theta}(\theta) P_{\theta}(\theta)=\int_{0}^{\infty} f_{t} P\left(f_{t}, \theta\right) d f_{t},
$$

where $\left\langle f_{t}\right\rangle_{\theta}(\theta)$ is the average friction force along the direction $\theta$ and $P_{\theta}(\theta)$ is the probability density of contact normal orientations. Both $P_{\theta}$ and $\left\langle f_{t}\right\rangle_{\theta}$ are $\pi$ periodic since the contact normals have no intrinsic polarity. Since the average friction force vanishes, we have

$$
0=\left\langle f_{t}\right\rangle=\int_{0}^{\pi}\left\langle f_{t}\right\rangle_{\theta}(\theta) P_{\theta}(\theta) d \theta .
$$

This equation implies that the functions $\left\langle f_{t}\right\rangle_{\theta}(\theta)$ and $P_{\theta}(\theta)$ are orthonormal.

Given the $\pi$ periodicity of the above functions, they can be Fourier-expanded, and at lowest order we get the following approximation, which satisfies the orthonormal condition,

$$
\begin{aligned}
P_{\theta}(\theta) & =\frac{1}{\pi}\left\{1+a_{c} \cos 2\left(\theta-\theta_{c}\right)\right\}, \\
\left\langle f_{t}\right\rangle_{\theta} & =\left\langle f_{n}\right\rangle a_{t} \sin 2\left(\theta-\theta_{t}\right),
\end{aligned}
$$

where $a_{c}$ represents the fabric anisotropy of contact normals, $a_{t}$ is the anisotropy of friction forces, $\theta_{c}$ is the privileged direction of contact normals, and $\theta_{t}$ is the reference direction for friction forces. The normalization of $\left\langle f_{t}\right\rangle_{\theta}$ by the average normal force $\left\langle f_{n}\right\rangle$ is necessary for the definition of a dimensionless anisotropy $a_{t}$. Figure 15 shows polar diagrams of the simulation data for $\left\langle f_{t}\right\rangle_{\theta}(\theta)$ and $P_{\theta}(\theta)$ together with the Fourier fits in the residual state. The fits are excellent. We see that $\theta_{c} \simeq \theta_{t}=\pi / 2$ coincides with the principal stress direction $\theta_{\sigma}=\pi / 2$.

In this way, we define a friction mobilization function:

$$
M_{f}(\theta) \equiv \frac{\left\langle f_{t}\right\rangle_{\theta}}{\mu\left\langle f_{n}\right\rangle}=\frac{a_{t}}{\mu} \sin 2\left(\theta-\theta_{t}\right) .
$$

This function has two modes along the directions $\theta_{t} \pm \pi / 4$ and the ratio $a_{t} / \mu$ is simply their amplitude. This amplitude can therefore be used as a descriptor of friction mobilization. Note that a descriptor of friction mobilization $I_{f}^{\prime}$ can also be defined by integrating the absolute value of $M_{f}(\theta)$ in the range $[0, \pi]$. This leads to the relation $I_{f}^{\prime}=2 a_{t} / \mu$. This definition is similar to that of $I_{f}$ with the difference that Eq. (16) is the average of the ratio of tangential and normal forces, whereas $I_{f}^{\prime}$ involves the ratio of the averages. Hence, we have $I_{f}^{\prime}=2 a_{t} / \mu \leqslant I_{f}$.

The friction mobilization increases from zero in the isotropic state with shear strain and its value in the residual state depends on the nature of the material. In Fig. 16, $I_{f}^{*}$,

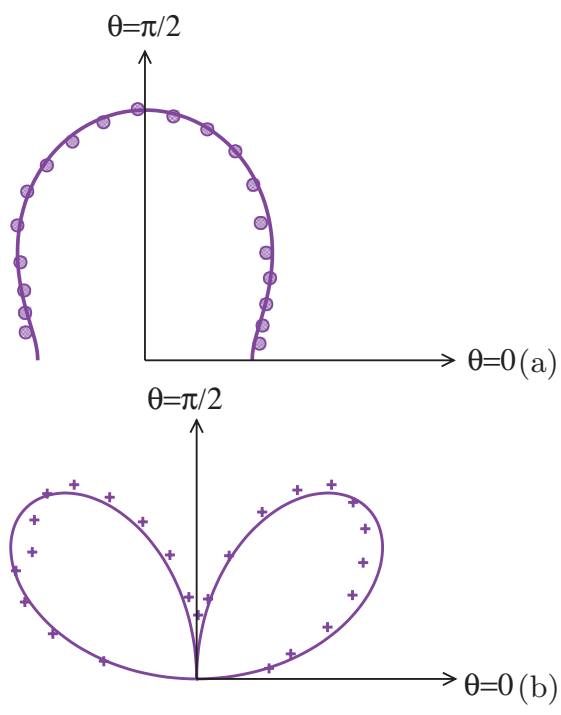

FIG. 15. (Color online) Polar diagrams of the probability density of contact orientations $\theta$ (a) and friction mobilization function (b) in the residual state for $\eta=0.7$. The solid lines are leading-order Fourier fits.

representing the residual-state value of $I_{f}$, is displayed as a function of $\eta . I_{f}^{*}$ increases linearly with $\eta$ from $\simeq 0.45$ for disks and saturates to $\simeq 0.62$ above $\eta=0.4$ in the same manner as the shear strength (Fig. 4). This high correlation between the shear strength and friction mobilization at increasing level of shape nonconvexity indicates that particle shape-dependence of shear strength is at least partially mediated by friction mobilization.

Figure 17 displays $a_{t}^{*} / \mu$ and $a_{c}^{*}$, representing the residualstate values of $a_{t} / \mu$ and $a_{c}$, as a function of $\eta . a_{t}^{*} / \mu$ shows the same trend as $I_{f}^{*}$ with values only slightly below $0.5 I_{f}^{*}$. In the same way, $a_{c}^{*}$ increases from 0.3 to a steady value 0.4 beyond $\eta \simeq 0.3$. Hence, the two descriptors of friction mobilization are almost equivalent and the shear strength is correlated with both friction mobilization and fabric anisotropy. This point is discussed in more detail in Sec. VII.

Another aspect of friction mobilization is the proportion $S$ of sliding contacts in steady shearing [39]. Figure 18 displays $S^{*}$ in the residual state as a function of $\eta$. $S^{*}$ varies about 0.13 at $\eta=0$ to about 0.28 for $\eta>0.4$. Hence, the number

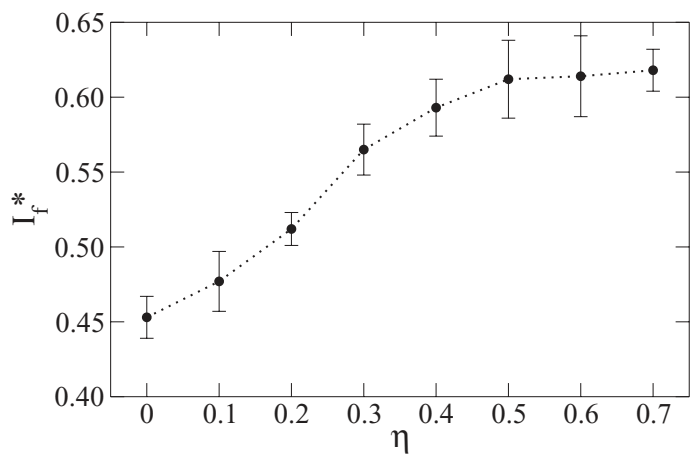

FIG. 16. Friction mobilization $I_{f}^{*}$ in the residual state as a function of nonconvexity. Error bars represent the standard deviation of the data. 

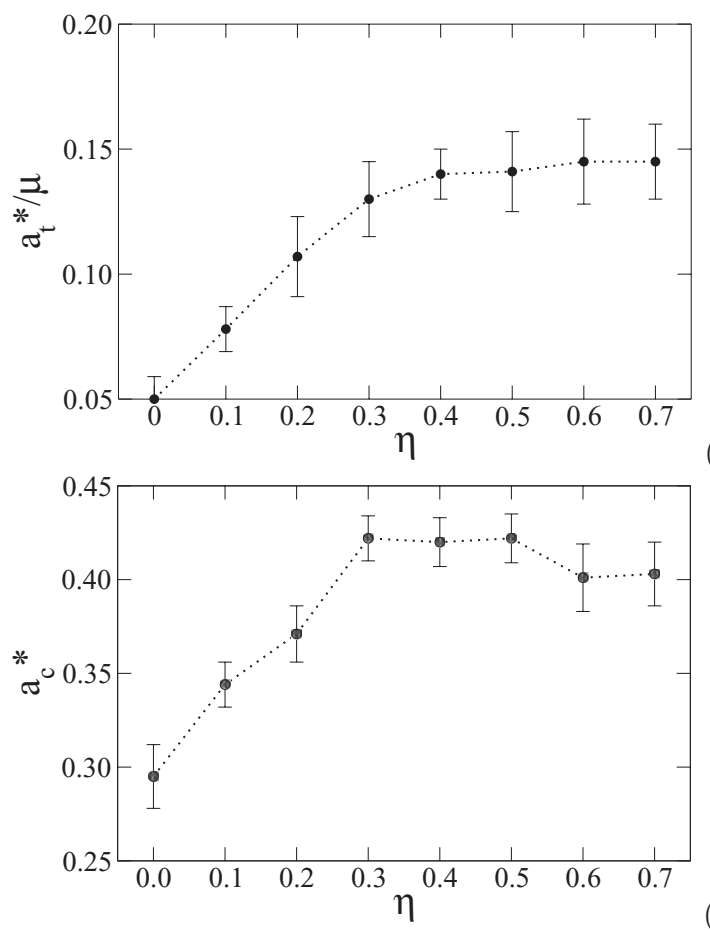

(a)

FIG. 17. Friction mobilization $a_{t}^{*} / \mu$ (a) and fabric anisotropy $a_{c}^{*}$ (b) in the residual state as a function of nonconvexity. Error bars represent the standard deviation of the data.

of sliding contacts follows the average friction mobilization. The fact that none is affected by increasing the nonconvexity of the aggregates beyond $\eta=0.5$ is important information for understanding the saturation of shear strength. Another key information is that the sliding contacts are unevenly distributed among simple, double-simple, double, and triple contacts, as shown in Fig. 19. Only a weak number of triple and double contacts are sliding, whereas the proportion of sliding double-simple contacts increases with $\eta$ at the expense of simple contacts. Hence, in contrast to $S_{D}^{*}$, which remains far below $S_{D S}^{*}$, the proportion $K_{D}^{*}$ of double contacts increases much faster than the proportion $K_{D S}^{*}$ of double-simple contacts (Fig. 11).

This differentiation between the roles played by the double and double-simple contacts is maybe the best demonstration

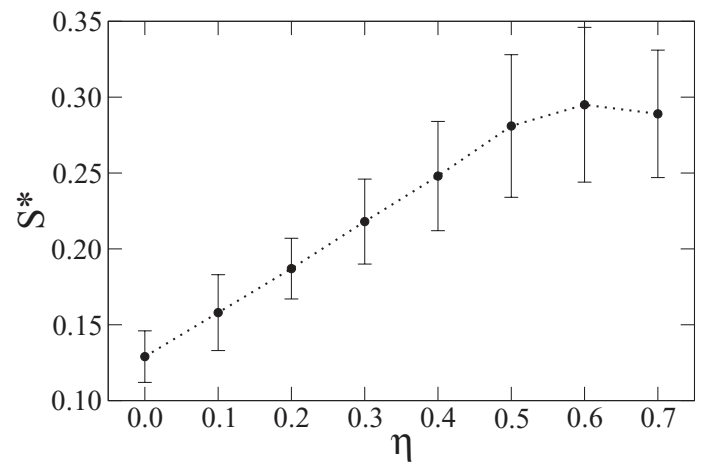

FIG. 18. Proportion of sliding contacts as a function of $\eta$ in the residual state. Error bars show the standard deviation in the residual state.

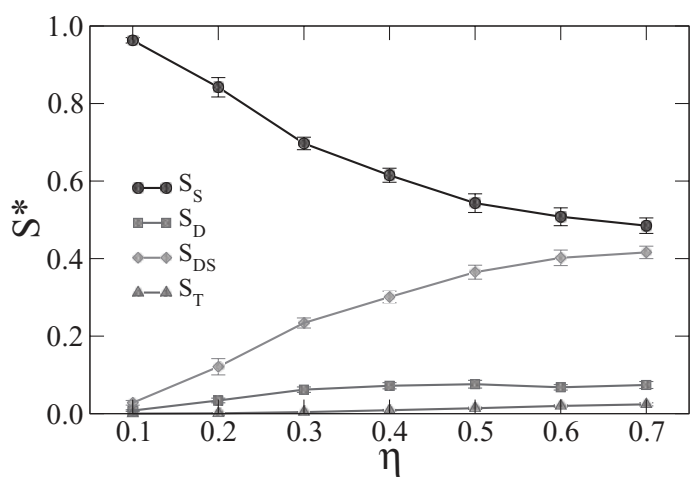

FIG. 19. (Color online) Proportions of sliding contacts as a function of $\eta$ in the residual state for different contact types between aggregates. Error bars show the standard deviation in the residual state.

of interlocking of the aggregates with the evident effect of freezing the relative motion and sliding at the double and triple contacts. As a result, frictional dissipation between the most nonconvex aggregates occurs preferentially at the simple and double-simple contacts. This enhanced interlocking has the same effect as cohesion forces in a cohesive granular material: As the aggregates are increasingly interlocked, they move together in the form of rigid clusters composed of several aggregates, giving rise to larger dilatation and thus lower packing fractions. This mechanism explains the lower values of packing fraction at high nonconvexity reached in the residual state compared to the isotropic state, where friction plays no role (see Fig. 6).

\section{FABRIC AND FORCE ANISOTROPIES}

The fabric anisotropy $a_{c}$ and the tangential force anisotropy $a_{t}$ were introduced in the last section in connection with friction mobilization. A similar definition can be applied to the angular dependence of the average normal forces [53]. We consider the joint probability density $P\left(f_{n}, \boldsymbol{n}\right)$ of the normal force $f_{n}$ and its orientation $\boldsymbol{n}$. In 2D, the vector $\boldsymbol{n}=(\cos \theta, \sin \theta)$ is parametrized by $\theta$ and we have

$$
\left\langle f_{n}\right\rangle_{\theta}(\theta) P_{\theta}(\theta)=\int_{0}^{\infty} f_{n} P\left(f_{n}, \theta\right) d f_{n},
$$

where $\left\langle f_{n}\right\rangle_{\theta}(\theta)$ is the average normal force along the direction $\theta$. The average normal force is

$$
\left\langle f_{n}\right\rangle=\int_{0}^{\pi}\left\langle f_{n}\right\rangle_{\theta}(\theta) P_{\theta}(\theta) d \theta .
$$

The Fourier expansion of $\left\langle f_{n}\right\rangle_{\theta}(\theta)$ at leading order together with the condition (22) yield

$$
\left\langle f_{n}\right\rangle_{\theta}=\left\langle f_{n}\right\rangle\left\{1+a_{n} \cos 2\left(\theta-\theta_{n}\right)\right\},
$$

where $a_{n}$ is the anisotropy of normal forces with privileged direction $\theta_{n}$. This form is well fit to the data as shown in Fig. 20 in the residual state for $\eta=0$.7. We also see that $\theta_{n}$ coincides with the principal stress direction $\theta_{\sigma}=\pi / 2$.

The residual-state value of normal-force anisotropy $a_{n}^{*}$ is displayed in Fig. 21 as a function of $\eta$. It increases with $\eta$ and, up to fluctuations, saturates to a value nearly independent of $\eta$. 


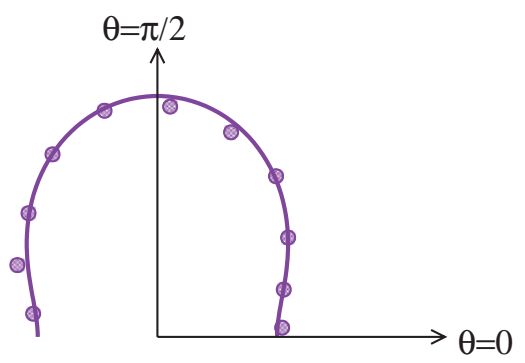

FIG. 20. (Color online) Polar diagram of the angular average of normal forces in the residual state for $\eta=0.7$. The solid line is the leading-order Fourier fit.

This is consistent with the behavior of friction mobilization and fabric anisotropy. Since $a_{n}$ represents the anisotropic structure of the force chains, its saturation indicates that nonconvexity has no influence on the force chains beyond $\eta \simeq 0.4$.

The force and fabric anisotropies are at the origin of shear strength in granular materials. Indeed, the expression of stress tensor (5) together with the Fourier expansions (20) and (24) lead to the following relation in the residual state [53]:

$$
\sin \varphi^{*}=\frac{q^{*}}{p} \simeq \frac{1}{2}\left(a_{c}^{*}+a_{n}^{*}+a_{t}^{*}\right) .
$$

This expression is based on the following assumptions, which are satisfied with a good approximation in the residual state: (1) The contact forces and branch-vector lengths are weakly correlated; (2) the reference directions coincide with the major principal stress direction, $\theta_{c}=\theta_{t}=\theta_{n}=\theta_{\sigma}$; (3) the cross products among the three anisotropies are negligible. The values of shear strength given by this expression from the anisotropies predict correctly the measured shear strength as observed in Fig. 22 for all values of $\eta$ in the residual state. Equation (25) allows us to evaluate the relative weights of fabric anisotropy, force chains, and friction mobilization with regard to shear strength. From Figs. 17 and 21 we see that $a_{t}^{*}<a_{n}^{*}<a_{c}^{*}$, so that the largest and smallest contributions are due to fabric anisotropy and friction mobilization, respectively. On the other hand, the saturation of shear strength beyond $\eta \simeq 0.4$ is mainly due to the saturation of friction mobilization and fabric anisotropy.

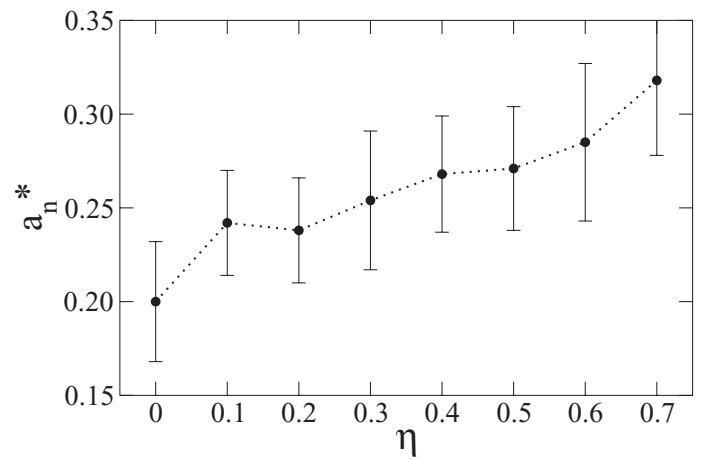

FIG. 21. Normal-force anisotropy $a_{n}^{*}$ in the residual state as a function of nonconvexity. Error bars represent the standard deviation of the data.

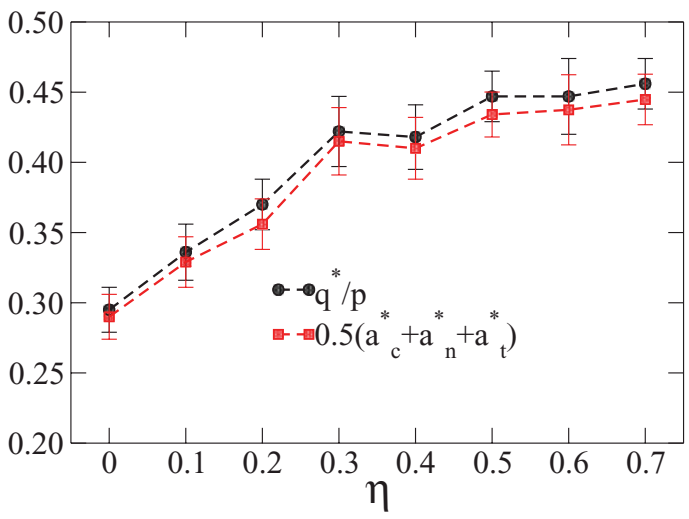

FIG. 22. (Color online) Normalized shear stress $q^{*} / p$ in the residual state as a function of $\eta$ (black line) with the harmonic approximation (red line) given by Eq. (25). Error bars represent the standard deviation of the data.

\section{A MODEL OF PACKING FRACTION}

The unmonotonic evolution of the packing fraction $\rho$ with the nonconvexity parameter $\eta$ is intriguing as it contrasts with a common intuition that the packing fraction of nonconvex particles should decrease as a result of interlocking. We would like to show here that the unmonotonic evolution of $\rho$ with $\eta$ may be understood from the very definition of $\eta$ and by discerning at least two different types of pores between the aggregates.

Let us consider the mean particle volume $V_{p}$ and the mean free volume $V_{f}$ defined as the mean pore volume per particle, and both normalized by the reference volume $\pi R^{2}$. We assume that the volume fractions in both initial and residual states are homogeneously distributed so that the total packing fraction results from particle-scale interactions rather than large-scale strain localization. This condition is nearly satisfied for the initial state due to the preparation process and for the residual state as a result of long shearing (see Sec. III B). The packing fraction is defined by

$$
\rho=\frac{V_{p}}{V_{p}+V_{f}} .
$$

Given the normalization, we have $V_{p}=1$ for $\eta=0$. The volume $1-V_{p}$ represents the mean self-porosity of a particle, whereas $V_{f}-\left(1-V_{p}\right)$ is the remaining free volume of the interstitial space of three aggregates or more. As $\eta$ increases, $V_{p}$ declines and self-porosity increases. According to Eq. (26), if the void ratio $e=V_{f} / V_{p}$ were an increasing function of $\eta$, then the packing fraction would decrease with $\eta$. Since this is not what we observe at low $\eta, e$ is expected to decrease as $\eta$ varies from 0 to 0.2 .

The variation of $V_{p}$ with $\eta$ can easily be calculated for the geometry of aggregates. We have

$$
V_{p}=\frac{r^{2}}{\pi}\left\{\frac{5 \pi}{2}-3 \cos ^{-1}(\lambda)+\sqrt{3} \lambda^{2}+3 \lambda \sqrt{1-\lambda^{2}}\right\},
$$

with

$$
r=\left\{1+\frac{2 \sqrt{3}}{3} \lambda\right\}^{-1}
$$


where we have set $R=1$. The parameter $\lambda$ can be expressed as a function of $\eta$ by inverting Eq. (2).

The free volume $V_{f}$ can be approximated as a sum of four terms:

$$
V_{f}(\eta)=V_{f}(0)+\Delta V_{f}^{(1)}(\eta)-\Delta V_{f}^{(2)}(\eta)-\Delta V_{f}^{(3)}(\eta) .
$$

$V_{f}(0)$ is the free volume at $\eta=0 . \Delta V_{f}^{(1)}$ is the gain of free volume due to the increase of self-porosity with $\eta$, simply given by

$$
\Delta V_{f}^{(1)}=1-V_{p},
$$

whereas $\Delta V_{f}^{(2)}$ is the loss of free volume due to interlocking, which implies the overlap of their self-porosities. The distance between the aggregates is reduced as the concave borders of an aggregate are partially filled by the convex parts of a neighboring aggregate [see Fig. 23(a)]. As a first approximation, we assume that $\Delta V_{f}^{(2)}$ varies proportionally to the self-porosity:

$$
\Delta V_{f}^{(2)}=f(\eta)\left(1-V_{p}\right),
$$

where $f(\eta)$ is the filling rate depending on $\eta$. The net volume change $\Delta V_{f}^{(1)}-\Delta V_{f}^{(2)}=\{1-f(\eta)\}\left(1-V_{p}\right)$ is always positive (increasing free volume) since, due to steric exclusions, the interlocking can never fully compensate the increase of self-porosity with $\eta$. In other words, the extra free volume created by the increasing concavity of the particle borders cannot be filled by the convex borders of neighboring particles. Moreover, an increasing number of large pores appear between the nonconvex borders of the aggregates [see Fig. 23(b)]. For this reason, the combination of self-porosity and interlocking cannot explain the increase of $\rho$ with $\eta$ in the range $[0,0.2]$. We also expect that $f(\eta)$ is a decreasing function of $\eta$ due to increasing angular hindrance between particles as their nonconvexity becomes larger. We assume here a linear decreasing function:

$$
f(\eta)=\alpha(1-\gamma \eta)
$$

The last term $\Delta V_{f}^{(3)}$ represents the variation of free volume as a result of the size variation of pores involving only the convex borders of the particles [see Fig. 23(c)]. The radius of curvature of the convex borders is the radius $r$ of the disks,

(b)

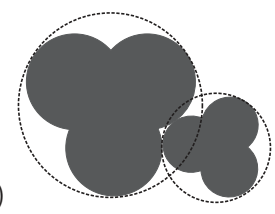

(a)

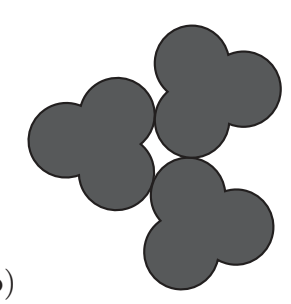

(c)

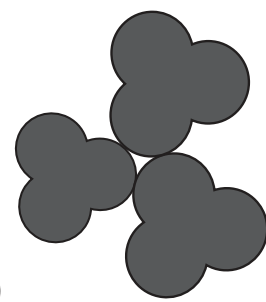

FIG. 23. (a) Overlap between the self-porosities of two aggregates. (b) A pore involving at least one nonconvex border. (c) A pore between the convex borders of aggregates.

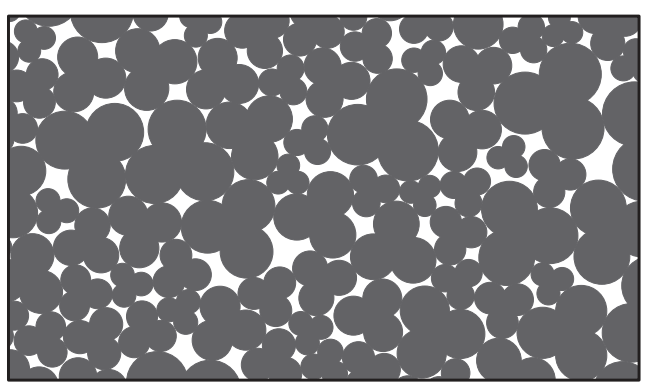

FIG. 24. A snapshot of a portion of the isotropic sample for $\eta=$ 0.5

which declines for a fixed value $R=1$ of the circumscribing circle. Hence, the volume of a pore between the convex borders of three or more particles decreases as $-r^{2}$. Therefore, given that $r(\eta=0)=R=1$, we set

$$
\Delta V_{f}^{(3)}=\beta\left(1-r^{2}\right),
$$

where $\beta$ is assumed to be a constant. In contrast to $\Delta V_{f}^{(1)}-$ $\Delta V_{f}^{(2)}, \Delta V_{f}^{(3)}$ is a decreasing function of $\eta$ and it explains the increase of $\rho$ with $\eta$ in the range $[0,0.2]$. The reduction of the pores composed of convex borders is clearly observed in Fig. 24, where a snapshot of the isotropic sample is displayed for $\eta=0.5$. We observe many small pores between the convex borders of the aggregates as well as large pores involving concave borders.

Inserting the expression of $V_{f}$ from Eq. (29) in Eq. (26) together with Eqs. (30), (31), and (33), we arrive at the following expression for packing fraction:

$$
\frac{\rho(\eta)}{\rho(0)}=\frac{V_{p}}{1-\alpha \rho(0)(1-\gamma \eta)\left(1-V_{p}\right)-\beta \rho(0)\left(1-r^{2}\right)} .
$$

Given the expressions of $V_{p}$ in Eq. (27) and $r$ in Eq. (28) as a function of $\lambda(\eta)$ obtained from the inversion of Eqs. (2) and (34) provides an excellent fit for our data points by adjusting the values of the parameters from the position of the peak and the packing fraction at $\eta=0.7$ for $\varepsilon_{q}=0$ and $\varepsilon_{q}=0.5$, as shown in Fig. 25. The best fit is obtained at $\varepsilon_{q}=0$ for

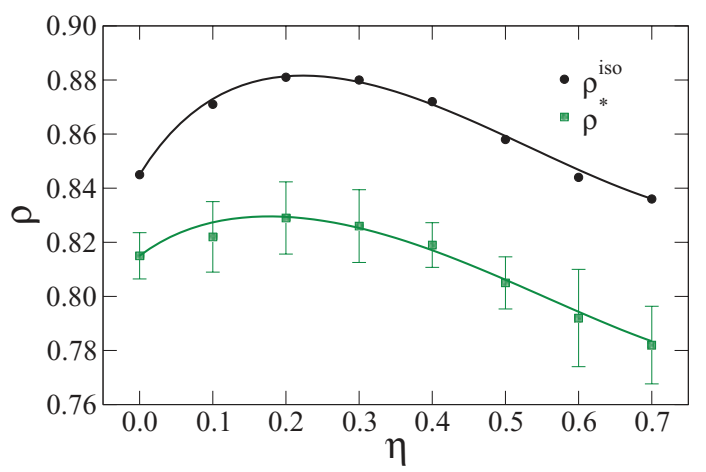

FIG. 25. (Color online) Packing fraction $\rho$ as a function of $\eta$ in the initial isotropic state and in residual state (at $\varepsilon_{q}=0.5$ ). The symbols are the simulation data, whereas the solid lines are analytical fits by Eq. (34). The error bars represent standard deviation in the residual state. 
$\alpha=0.87, \beta=0.17$, and $\gamma=0.17$, and at $\varepsilon_{q}=0.5$ for $\alpha=1$, $\beta=0.11$, and $\gamma=0.18$.

The main assumption of the simple model introduced above is that the particle shape is inside a circumscribing circle of radius $R$ and it occupies a portion of the total surface $\pi R^{2}$ of the circle with a central part which is fully inscribed in a circle of radius $(1-\eta) R$. This model can therefore be applied to arbitrary shapes which can be described by similar parameters, and we expect a similar unmonotonic dependence of the packing fraction with respect to $\eta$.

\section{DISCUSSION AND CONCLUDING REMARKS}

Large packings of nonconvex aggregates were simulated and analyzed in this work with the aim of quantifying the effect of nonconvex particle shape on the behavior of granular materials. The aggregates are of a rather simple shape characterized by a threefold rotational symmetry and a single nonconvexity parameter $\eta$ varying from 0 (corresponding to a disk) to 0.73 (corresponding to a trimer of mutually contacting disks). The shape dependence appears to be rather nontrivial. One would expect the shear strength to increase with $\eta$ as a result of interlocking between aggregates. We do observe a linear increase of the shear strength, measured by the internal angle of friction in the residual state, but it saturates to a value independent of $\eta$. It is also remarkable that the packing fraction varies unmonotonically with nonconvexity, a feature that seems to be shared with other shapes described by deviation from circular shape.

The saturation of shear strength with nonconvexity recalls a similar effect observed when the friction coefficient is increased between particles in a granular packing [54]. The internal angle of friction in a packing of circular particles increases with friction coefficient, but tends to a constant value that is no more affected by further increase of the friction coefficient. This effect is attributed to the exhaustion of sliding contacts and a self-organization of the particle motions favoring rolling contacts, which do not dissipate energy. A similar picture emerges also from our analysis of the multiple contacts between aggregates. Indeed, an increasing number of aggregates are interlocked so that most double and triple contacts between aggregates are nonsliding. The aggregates move in clusters with relative sliding and rolling localized mainly at the simple and double-simple contacts. At the same time, larger pores occur due to this "clustered" motion of the aggregates and the packing fraction tends to decline. This frictional clustering is therefore a general mechanism, which provides an internal compensation mechanism in conjugation with rolling and free volume change that allow the system to keep a constant level of dissipation.

Another interesting feature of the packings of aggregates is that the connectivity of the aggregates does not follow the packing fraction. The effect of increasing nonconvexity is expressed by an increasing number of multiple contacts with essentially the same number of contact neighbors, whereas the packing fraction increases with $\eta$ before decreasing to a rather low level. This means that the packing fraction is not a good variable for the plastic behavior of granular materials composed of nonconvex particles. This should not come as a surprise since the expression of stress tensor does not involve the packing fraction. The internal variables exhibited by a harmonic decomposition of the stress tensor are the fabric anisotropy, normal-force anisotropy, and friction mobilization, formulated in terms of the friction force anisotropy. All these anisotropies enter the expression of the shear strength and follow nearly the same trend.

We would like also to underline here the specificity of the isostatic state, which shows a basically invariant structure with regard to the nonconvexity of the aggregates. The coordination number, connectivity, proportions of different types of contact between aggregates and probability densities of forces are independent of nonconvexity. Nevertheless, the variation of packing fraction is unmonotonic with $\eta$ in the isostatic state, too. This is a major argument in favor of the phenomenological model introduced in this paper for packing fraction with ingredients based on the particle shape rather than a particular state of the material.

Further work is presently under way with more complex nonconvex geometries and in $3 \mathrm{D}$ in order to evaluate the robustness of the results presented in this paper. The analysis presented in this paper may be generalized to other particle shapes described by a parameter similar to $\eta$, which essentially refers to a deviation from circular shape. This idea was investigated within a benchmark by a collaborative group for different particle shapes. The results of this work will be presented elsewhere.
[1] H. Jaeger and S. Nagel, Rev. Mod. Phys. 68, 1259 (1996).

[2] B. Cambou and A. Danescu, in Multiscale Techniques for Granular Materials, Chap. 3 (Wiley, New York, 2009), pp. 101-145.

[3] F. Radjai, H. Troadec, and S. Roux, in Granular Materials: Fundamentals and Applications, edited by S. Antony, W. Hoyle, and Y. Ding (Royal Society of Cambridge, Cambridge, 2004), pp. 157-184.

[4] H. Ouadfel and L. Rothenburg, Mech. Mater. 33, 201 (2001).

[5] C. Nouguier-Lehon, B. Cambou, and E. Vincens, Int. J. Numer. Anal. Methods Geomech. 27, 1207 (2003).

[6] E. Azema, F. Radjai, and G. Saussine, Mech. Mater. 41, 729 (2009).
[7] A. Donev, F. H. Stillinger, P. M. Chaikin, and S. Torquato, Phys. Rev. Lett. 92, 255506 (2004).

[8] A. Donev, I. Cisse, D. Sachs, E. Variano, F. H. Stillinger, R. Connelly, S. Torquato, and P. M. Chaikin, Science 303, 990 (2004).

[9] W. N. Man, A. Donev, F. H. Stillinger, M. T. Sullivan, W. B. Russel, D. Heeger, S. Inati, S. Torquato, and P. M. Chaikin, Phys. Rev. Lett. 94, 198001 (2005).

[10] E. Azema and F. Radjai, Phys. Rev. E 81, 051304 (2010).

[11] S. Torquato and Y. Jiao, Phys. Rev. E 82, 051304 (2010).

[12] U. Agarwal and F. A. Escobedo, Nat. Mater. 10, 230 (2011).

[13] E. Azema, F. Radjai, R. Peyroux, and G. Saussine, Phys. Rev. E 76, 011301 (2007). 
[14] J. Fourcade, P. Sornay, F. Sudreau, and P. Papet, Powder Metall. 49, 125 (2006).

[15] F. Radjai and V. Richefeu, Mech. Mater. 41, 715 (2009).

[16] M. Jean, Discrete-element Modeling of Granular Materials, Chap. Contact Dynamics Method (Wiley, New York, 2011), pp. 27-65.

[17] J. Mitchell and K. Soga, Fundamentals of Soil Behavior (Wiley, New York, NY, 2005)

[18] S. Blott and K. Pye, Sedimentology 55, 31 (2008).

[19] J. J. Moreau, Eur. J. Mech., A/Solids 13, 93 (1994).

[20] M. Jean, Comput. Methods Appl. Mech. Eng. 177, 235 (1999).

[21] P. A. Cundall and O. D. L. Strack, Geotechnique 29, 47 (1979).

[22] M. P. Allen and D. J. Tildesley, Computer Simulation of Liquids (Oxford University Press, Oxford, 1987).

[23] H. J. Herrmann, Chaos, Solitons Fractals 6, 203 (1995).

[24] S. McNamara, Discrete-element Modeling of Granular Materials, Chap. Molecular Dynamics Method (Wiley-ISTE, New York, 2011), pp. 1-26.

[25] F. Radjai and S. Roux, Phys. Rev. E 51, 6177 (1995).

[26] F. Radjai, Physics of Dry Granular Media, Chap. Multicontacts Dynamics (Kluwer Academic, Dordrecht/Boston/London, 1997), p. 305.

[27] F. Radjai, S. Roux, and J. J. Moreau, Chaos 9, 544 (1999).

[28] J. Lanier and M. Jean, Powder Technol. 109, 206 (2000).

[29] F. Radjai and S. Roux, in The Physics of Granular Media, edited by H. Hinrichsen and D. E. Wolf (Wiley-VCH, Weinheim, 2004), pp. 165-186.

[30] F. Dubois, Discrete-element Modeling of Granular Materials, Chap. Numerical Modeling of Granular Media Composed of Polyhedral Particles (Wiley-ISTE, New York, 2011), pp. 233-262.

[31] I. Bratberg, F. Radjai, and A. Hansen, Phys. Rev. E 66, 031303 (2002).

[32] A. Taboada, K. J. Chang, F. Radjai, and F. Bouchette, J. Geophys. Res. 110, 1 (2005).

[33] C. Voivret, F. Radjai, J. Y. Delenne, and M. S. El Youssoufi, Phys. Rev. E 76, 021301 (2007).
[34] C. Voivret, Ph.D. thesis, Université de Montpellier 2, 2008.

[35] E. Clement, Colloid Interface Sci. 4, 294 (1999).

[36] GDR-MiDi, Eur. Phys. J. E 14, 341 (2004).

[37] L. Rothenburg and A. P. S. Selvadurai, in Mechanics of Structured Media, edited by A. P. S. Selvadurai (Elsevier, Amsterdam, 1981), pp. 469-486.

[38] J. J. Moreau, in Friction, Arching, Contact Dynamics, edited by D. E. Wolf and P. Grassberger (World Scientific, Singapore, 1997), pp. 233-247.

[39] L. Staron and F. Radjai, Phys. Rev. E 72, 041308 (2005).

[40] A. Donev, R. Connelly, F. H. Stillinger, and S. Torquato, Phys. Rev. E 75, 051304 (2007).

[41] I. Agnolin and J.-N. Roux, Phys. Rev. E 76, 061302 (2007).

[42] I. Agnolin and J.-N. Roux, Phys. Rev. E 76, 061303 (2007).

[43] I. Agnolin and J.-N. Roux, Phys. Rev. E 76, 061304 (2007).

[44] C. Liu, S. R. Nagel, D. A. Schecter, S. N. Coppersmith, S. Majumdar, O. Narayan, and T. A. Witten, Science 269, 513 (1995).

[45] S. N. Coppersmith, C.-h. Liu, S. Majumdar, O. Narayan, and T. A. Witten, Phys. Rev. E 53, 4673 (1996).

[46] F. Radjai, M. Jean, J.-J. Moreau, and S. Roux, Phys. Rev. Lett. 77, 274 (1996).

[47] P. T. Metzger, Phys. Rev. E 70, 051303 (2004).

[48] T. S. Majmudar and R. P. Behringer, Nature (London) 435, 1079 (2005).

[49] J. H. Snoeijer, W. G. Ellenbroek, T. J. H. Vlugt, and M. van Hecke, Phys. Rev. Lett. 96, 098001 (2006).

[50] V. Richefeu, M. S. El Youssoufi, E. Azéma, and F. Radjai, Powder Technol. 190, 258 (2009).

[51] V. Topin, F. Radjai, and J.-Y. Delenne, Phys. Rev. E 79, 051302 (2009).

[52] B. P. Tighe, J. H. Snoeijer, T. J. H. Vlugt, and M. van Hecke, Soft Matter 6, 2908 (2010).

[53] L. Rothenburg and R. J. Bathurst, Geotechnique 39, 601 (1989).

[54] N. Estrada, A. Taboada, and F. Radjai, Phys. Rev. E 78, 021301 (2008). 